\title{
Reconhecendo a sobrecarga e a qualidade de vida de cuidadores familiares de idosos frágeis
}

\author{
Recognizing burden and quality of life of family caregivers of frail elderly
}

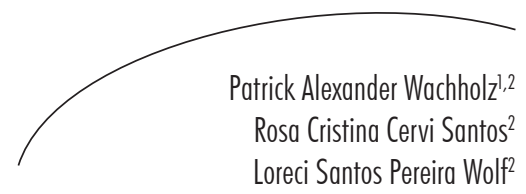

\section{Resumo}

Objetivos: Analisar as correlações entre o grau de dependência funcional de idosos residentes na comunidade, o nível de sobrecarga relacionada ao cuidado e a percepção de qualidade de vida dos cuidadores familiares desses idosos. Métodos: Estudo observacional, descritivo e analítico, com amostragem não probabilística selecionada por conveniência entre dezembro de 2008 e maio de 2009 na área urbana de Curitiba e Colombo, estado do Paraná. As entrevistas foram aplicadas aos cuidadores, empregando-se: inquérito sociodemográfico; avaliação funcional do idoso; avaliação da sobrecarga (Zarit-BurdenInterview) e qualidade de vida (WHOQOL-Bref). Utilizaram-se os coeficientes de Spearman, de Mann-Whitney e Kruskal Wallis para avaliar as correlações entre as escalas e destas com variáveis sociodemográficas. Análise bivariada identificou quais variáveis se correlacionariam à sobrecarga, incluídas as significativas em modelo de regressão linear múltipla. Resultados: Foram entrevistados 45 cuidadores, predominando mulheres $(91,11 \%)$ com elevada escolaridade, assistindo idosos funcionalmente dependentes (66,77\%). Percebeu-se sobrecarga moderada/severa em 75,55\% da amostra. Observouse correlação entre dependência funcional e maior sobrecarga no cuidador $(r=-0,281$, $\mathrm{p}=0,013)$ e pior percepção de qualidade de vida. A regressão linear múltipla identificou forte associação entre sobrecarga relacionada ao cuidado e o domínio psicológico do WHOQOL-bref e o tempo como cuidador $\left(\mathrm{R}^{2}=0,58, \mathrm{p}<0,001\right)$. Conclusão: Em amostra de cuidadores familiares, foram identificadas correlações significativas entre menor nível de sobrecarga relacionada ao cuidado e melhores percepções de qualidade de vida, bem como associação entre maior grau de dependência do idoso assistido e maior sobrecarga e qualidade de vida menos satisfatórias.

\footnotetext{
Programa de Pós-Graduação em Saúde Coletiva, Faculdade de Medicina de Botucatu. Universidade Estadual Paulista Julio de Mesquita Filho. Botucatu, SP, Brasil.

2 Programa de Pós-graduação em Geriatria e Gerontologia Social. Universidade Positivo. Curitiba, PR, Brasil.
}

Palavras-chave: Idoso. Cuidadores. Qualidade de vida. Assistência Domiciliar. Pacientes Domiciliares. 


\section{Abstract}

Objective: This study aimed to analyze the correlation between the levels of functional dependence of elderly living in the community, the burden related to care and the perception of quality of life in familiar caregivers. Methods: This is an observational, descriptive and analytical study, using non probabilistic sampling selected by convenience in the period from December 2008 to May 2009, in the urban area of Curitiba and Colombo, state of Paraná, Brazil. Interviews were applied to caregivers, using demographic inquiry, functional evaluation of the aged, burden interview (ZaritBurden-Interview) and quality of life instrument (WHOQOL-Bref). Spearman, Mann-Whitney and Kruskal Wallis coefficients were used to analyze the correlations between instruments and socio-demographic variables. Bivariate analyses identified which variables correlate with burden, and the most significant were included in a multiple linear regression. Results: Forty-five caregivers were interviewed, mostly women $(91.11 \%)$ with high educational level attending dependent elderly $(66.77 \%)$. Moderate/severe burden was perceived in $75.55 \%$ of the sample. We found correlation between dependence, more severe burden in caregivers $(r=-0.281, \mathrm{p}=0.013)$ and worse perception of quality of life. The multiple linear regression identified strong association between burden related to care and psychological domain from WHOQOL-bref and time as caregiver $\left(\mathrm{R}^{2}=0.58, \mathrm{p}<0.001\right)$. Conclusion: In a sample of familiar caregivers, we identified correlations between lower burden related to care and better quality of life perceptions, as well as higher disability and less satisfactory quality of life perceptions.
Key words: Aged. Caregivers. Quality of Life. Home Nursing. Homebound Persons.

\section{INTRODUÇÃO}

A proporção de idosos brasileiros com alguma dificuldade para desempenhar as atividades básicas de vida diária é de aproximadamente $13,5 \%$, sendo mais frequente em mulheres e em pessoas acima dos oitenta anos. ${ }^{1}$ Nesse contexto, a disponibilização do auxílio de outra pessoa para tomar banho, vestir-se, alimentar-se e/ou fazer a própria higiene, por exemplo, torna-se imprescindível.

Conviver todos os dias com as necessidades e demandas de uma pessoa portadora de doenças crônicas pode ser uma experiência extremamente angustiante, especialmente se essa pessoa for um parente próximo, idoso ou portador de alguma incapacidade física ou cognitiva. ${ }^{2,3}$ Estudos norte-americanos estimam que pessoas com demência severa necessitam de 41,5 horas por semana de cuidados informais adicionais; 17,4 horas nos quadros demenciais moderados e 8,5 horas nos quadros leves, contrastando com 4,6 horas por semana nos idosos com cognição normal, o que representa um custo anual superior a U\$ 18 bilhões. ${ }^{4}$

$\mathrm{Na}$ literatura, define-se como cuidador a "pessoa que desempenha a função de ajudar pessoas dependentes, numa relação de proximidade física e afetiva". ${ }^{5}$ Segundo alguns autores, "cuidar" é um ato de vida, que tem por finalidade assegurar a manutenção e continuidade da existência humana, pertencendo à esfera das responsabilidades familiares executá-lo. ${ }^{5,6} \mathrm{Com}$ frequência, um idoso dependente e sua família contam com uma rede insuficiente e precária de saúde preventiva e serviços de reabilitação. Quando não há condições ou disponibilidade para contratar um profissional especialmente treinado para este fim, ou seja, um cuidador profissional, em geral um parente ou membro da família assume a função de cuidador. ${ }^{5}$

As tarefas atribuídas ao cuidador familiar agregam forte impacto em sua vida, pois muitas 
vezes são executadas sem orientação adequada, sem o suporte dos outros membros da família, e frequentemente alteram toda a rotina de vida anterior, ocupando a maior parte do dia. ${ }^{8}$ Por vezes, o familiar que desempenha o papel de cuidador pode acabar adoecendo em decorrência dessa função, ${ }^{6}$ ao acumular para si atividades relacionadas ao cuidado e as de sua própria rotina pessoal. ${ }^{9}$

A despeito da importância do cuidador familiar na saúde pública brasileira, a maioria deste contingente não dispõe de informações e mínimo suporte necessários à assistência, o que "constitui-se em fator de risco para a manutenção de sua qualidade de vida."10

A sobrecarga relacionada ao cuidado é um construto complexo, que compreende uma série de aspectos e consequências na vida dos cuidadores e suas famílias, ${ }^{3}$ e tem sido relacionada ao desenvolvimento de problemas físicos e fadiga, perda de equilíbrio financeiro e de relacionamentos familiares e ocupacionais (sobrecarga objetiva), ${ }^{3}$ bem como sintomas psiquiátricos, uso de medicamentos psicotrópicos, efeitos sociais e emocionais (sobrecarga subjetiva). ${ }^{3,11}$ A literatura sugere que os efeitos prejudiciais do cuidado estariam primariamente associados às incapacidades funcionais, sejam elas físicas, cognitivas ou comportamentais, oriundas dos indivíduos assistidos pelo cuidador, e pela respectiva demanda por atenção e vigilância inerente a suas necessidades. ${ }^{2}$

Com frequência, os cuidadores também são afetados pelas experiências emocionais e psicológicas de sofrimento por que passam as pessoas que são por eles cuidadas, e os efeitos dessas inter-relações na qualidade de vida dos cuidadores ainda são apenas parcialmente compreendidos. . $^{2,3,10}$

O objetivo deste estudo foi analisar as correlações entre o grau de dependência funcional de idosos residentes na comunidade, o nível de sobrecarga relacionada ao cuidado e a percepção de qualidade de vida dos cuidadores familiares desses idosos.

\section{MÉTODOS}

Estudo com delineamento observacional, retrospectivo, descritivo e analítico, de abordagem quantitativa, realizado na área urbana das cidades de Curitiba e Colombo, PR. A amostragem foi não probabilística, selecionada por conveniência, entre os meses de dezembro de 2008 e maio de 2009. A amostra potencial do estudo foi composta por cuidadores familiares de idosos indicados por cinco profissionais de saúde (médicos e psicólogos) que atendiam em clínicas privadas, e do Sistema Único de Saúde (SUS) na área de abrangência do estudo, onde assistiam aos idosos ou aos próprios cuidadores.

Foram considerados como critérios de inclusão: ser cuidador familiar, ou seja, apresentar parentesco com o idoso assistido; idade igual ou superior a 30 anos e assistir ao idoso fragilizado por, no mínimo, dois anos completos na data de admissão no estudo. Por idoso fragilizado assumiu-se uma pessoa com 60 anos de idade ou mais, dependente da assistência ou supervisão de terceiros para a garantia da própria subsistência, independentemente da etiologia do déficit ou incapacidade (por exemplo, problemas físicos, prejuízo cognitivo, sofrimento psicossocial ou insuficiência financeira). Foram excluídos os cuidadores familiares que tivessem antecedentes de maustratos contra o idoso assistido, informados pelos profissionais que os recomendaram para o estudo quando questionados pelos pesquisadores após a indicação, ou que recebessem remuneração pelos serviços prestados como cuidador de idosos por parte da família, confirmados durante a entrevista.

Tendo sido os cuidadores indicados por um dos profissionais, um dos pesquisadores fazia o contato inicial por telefone, durante o qual eram informados os objetivos do estudo. Caso 
aceitassem conversar pessoalmente com os pesquisadores, recebiam, durante visita domiciliar agendada, todas as informações necessárias para compreenderem o estudo e assinavam o Termo de Consentimento Livre e Esclarecido.

Dos 62 cuidadores abordados no período do estudo, três foram excluídos (um por antecedente de maus-tratos e dois por receberem honorários dos familiares para realizarem o cuidado) e 14 não concordaram em participar do estudo. A amostra do estudo foi composta por 45 cuidadores familiares.

Em subsequente visita domiciliar, em ambiente privativo (de modo a não constranger o cuidador durante a entrevista e garantir o sigilo das informações compartilhadas), eram aplicados um questionário sociodemográfico e instrumentos traduzidos e validados para avaliação da sobrecarga associada ao cuidado (escala Zarit Burden Interview) $)^{12}$ e avaliação da percepção de qualidade de vida (WHOQOL-bref). ${ }^{13}$

O grau de dependência do idoso assistido foi determinado pela aplicação do índice de Barthel, ${ }^{14}$ segundo a percepção do cuidador entrevistado. Esse instrumento mensura a independência funcional na execução de dez tarefas da vida diária: alimentação, banho, vestuário, higiene pessoal, controle das eliminações intestinais, controle das eliminações vesicais, uso do vaso sanitário, passagem cadeira-cama, capacidade de deambulação e de subir degraus ou escadas. A pontuação varia de 0 a 100, cada tarefa sendo pontuada de acordo com a melhor capacidade de sua execução, mediante o uso de uma escala de Likert. Quanto maior a pontuação, melhor a capacidade funcional. $O$ instrumento foi traduzido e validado para aplicação no Brasil, ${ }^{14}$ tendo sido adotados valores superiores a 60 para indicar independência. ${ }^{15}$
Para avaliar a percepção de sobrecarga dos cuidadores, foi aplicada a escala Zarit Burden Interview (ZBI), composta por 22 itens que avaliam a relação entre o cuidador e idoso, a condição de saúde, o bem-estar psicológico, finanças e vida social. As respostas também são pontuadas por escala de Likert, de acordo com a presença ou intensidade de uma resposta afirmativa $(0$ = nunca, 1 = raramente, $2=$ algumas vezes, $3=$ frequentemente e $4=$ sempre). $\mathrm{O}$ escore total varia de 0 a 88, conferindo maiores percepções de sobrecarga a escores mais altos. $\mathrm{O}$ instrumento foi traduzido e validado para o português. ${ }^{12}$

Para a análise estatística, foi utilizada a classificação previamente publicada para os escores totais, ${ }^{7}$ determinando sobrecarga pequena para 0 a 20 pontos, sobrecarga moderada entre 21 e 40 pontos, moderada a severa para pontuação entre 41 e 60 , e sobrecarga severa para os cuidadores com pontuações entre 61 e o limite superior do ZBI.

A percepção de qualidade de vida (QV) entre os cuidadores foi avaliada por meio do questionário WHOQOL-bref, que contém 24 questões representativas de quatro facetas (domínios físico, psicológico, relações sociais e meio ambiente) e mais duas questões gerais, independentes dos escores dos domínios anteriormente citados, que compõem o Índice Geral de Qualidade de Vida (IGQV). A pontuação utilizada obedeceu ao escore de zero a 20; quanto maiores as pontuações, mais positivas são as percepções de qualidade de vida do indivíduo avaliado. O instrumento, iniciativa internacional chancelada pela Organização Mundial da Saúde, foi traduzido e validado pelo grupo WHOQOL Brasil. ${ }^{13}$

Os dados foram armazenados no software Microsoft Office Excel ${ }^{\circledR}$. A análise foi realizada utilizando-se o pacote estatístico do SPSS ${ }^{\circledR}$ 13.0, 
utilizando-se análise descritiva para interpretação das variáveis sociodemográficas. Foram aplicados: o coeficiente Alfa de Cronbach para confirmar a consistência interna dos instrumentos (índice de Barthel, ZBI e WHOQOL-bref) na amostra, considerando adequados valores superiores a 0,7; o coeficiente de correlação de Spearman para verificar a correlação entre os domínios do WHOQOL-bref, os escores do ZBI e a pontuação do índice de Barthel; e os coeficientes de MannWhitney e Kruskal Wallis para comparar os escores destes dois instrumentos com variáveis sociodemográficas de interesse, considerando significativos valores de $\mathrm{p} \leq 0,05$.

Para confirmar quais variáveis causaram maior impacto na sobrecarga relacionada ao cuidado, foi aplicada uma análise bivariada; as variáveis que demonstraram significância estatística nesta análise foram incluídas em modelo de regressão linear múltipla, adotando o escore total do ZBI (severidade da sobrecarga relacionada ao cuidado) como variável dependente.

O estudo foi conduzido respeitando-se a Resolução n.196/96 do Conselho Nacional de Saúde e as normas internacionais de pesquisa com seres humanos (Declaração de Helsinque), tendo adotado todos os procedimentos éticos previstos pelo Comitê Institucional de Ética em Pesquisa da Universidade Positivo, com aprovação sob o número CAAE 0121.0.094.000-08.

\section{RESULTADOS}

A amostra final foi composta por 45 cuidadores familiares, a maioria mulheres (91,1\%; n=41), com idade média de 55,13 \pm 5,79 anos (45-65). Cinquenta e três por cento $(\mathrm{n}=24)$ tinham nível superior de escolaridade; $70 \%(n=31)$ eram casados ou se encontravam em união consensual. Apenas 15,55\% $(\mathrm{n}=7)$ dos participantes não tinham filhos; os parentescos dos cuidadores com o idoso revelaram que $93,33 \%$ eram constituídos por filhos, enquanto os demais eram irmãos ou netos.

Vinte e seis cuidadores $(57,77 \%)$ informaram dedicar-se ao cuidado por dois a quatro anos, enquanto cinco entrevistados $(11,11 \%)$ assistiam ao idoso frágil há dez anos ou mais. A descrição pormenorizada das variáveis sociodemográficas e a caracterização do perfil do cuidado podem ser encontradas na tabela 1. 
Tabela 1 - Perfil sociodemográfico e características do cuidado em amostra de 45 cuidadores familiares. Curitiba-PR, 2008-2009.

\begin{tabular}{|c|c|}
\hline Variáveis & $\mathrm{n}(\%)$ \\
\hline \multicolumn{2}{|l|}{ Gênero } \\
\hline Masculino & $04(8,89)$ \\
\hline Feminino & $41(91,11)$ \\
\hline \multicolumn{2}{|l|}{ Idade (anos) } \\
\hline $45-50$ & $08(17,77)$ \\
\hline $51-55$ & $18(40,00)$ \\
\hline $56-60$ & $09(20,00)$ \\
\hline $61-65$ & $10(22,23)$ \\
\hline \multicolumn{2}{|l|}{ Escolaridade } \\
\hline Fundamental & $05(11,11)$ \\
\hline Médio & $16(35,55)$ \\
\hline Superior & $24(53,34)$ \\
\hline \multicolumn{2}{|l|}{ Estado civil } \\
\hline Casado/União consensual & $31(68,88)$ \\
\hline Solteiro & $06(13,33)$ \\
\hline Viúvo/Desquitado & $08(17,79)$ \\
\hline \multicolumn{2}{|l|}{ Tempo como cuidador } \\
\hline 2 a 4 anos & $26(57,77)$ \\
\hline 5 a 10 anos & $12(26,66)$ \\
\hline 10 anos ou mais & $05(11,11)$ \\
\hline NS/NR & $02(4,46)$ \\
\hline \multicolumn{2}{|l|}{ Tempo que mora junto com o idoso } \\
\hline 2 a 4 anos & $12(26,66)$ \\
\hline 5 a 10 anos & $08(17,77)$ \\
\hline 10 anos ou mais & $15(33,33)$ \\
\hline Casas separadas & $10(22,24)$ \\
\hline \multicolumn{2}{|l|}{ Por que é o cuidador? } \\
\hline Escolha própria & $19(42,22)$ \\
\hline Pessoa mais próxima & $12(26,66)$ \\
\hline Escolha do idoso & $04(8,88)$ \\
\hline Não ter outra pessoa disponível & $08(17,77)$ \\
\hline NR & $02(4,47)$ \\
\hline \multicolumn{2}{|c|}{ Relacionamento com o idoso anterior ao início do cuidado } \\
\hline Muito bom & $22(48,88)$ \\
\hline Bom & $16(35,55)$ \\
\hline Ruim & $03(6,66)$ \\
\hline Péssimo & $04(8,91)$ \\
\hline
\end{tabular}

$\mathrm{NS}=$ não sabe; $\mathrm{NR}=$ não respondeu. 
O escore médio de dependência funcional entre os idosos assistidos, avaliado por meio do

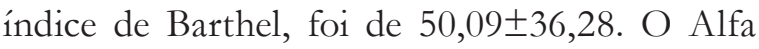
de Cronbach mensurado entre as dez questões do instrumento foi de 0,962 . A distribuição da capacidade funcional evidenciou que $30(66,77 \%)$ idosos eram dependentes, enquanto 15 (33,23\%) eram funcionalmente independentes.
O nível de sobrecarga médio mensurado pelo escore total do ZBI entre os cuidadores entrevistados foi de 35,18 \pm 16.20 (0-64). O Alfa de Cronbach entre as 22 questões do instrumento foi de 0,88 . A distribuição do nível de sobrecarga estimado pela análise dos escores totais dos participantes pode ser analisada na tabela 2 .

Tabela 2 - Percepção de sobrecarga entre cuidadores familiares, conforme o escore total da escala Zarit Burden Interview, em amostra de 45 cuidadores familiares. Curitiba-PR, 2008-2009.

\begin{tabular}{|c|c|c|}
\hline Classificação & Frequência & Percentual \\
\hline Pequena & 08 & 17,78 \\
\hline Moderada & 20 & 44,44 \\
\hline Moderada a severa & 14 & 31,11 \\
\hline Severa & 03 & 6,67 \\
\hline Total & 45 & 100,00 \\
\hline
\end{tabular}

Pertinente à avaliação da qualidade de vida, o escore médio do domínio físico do WHOQOLbref dos cuidadores foi de 13,98 $\pm 3,25$ (6,86-20). O domínio psicológico evidenciou pontuação

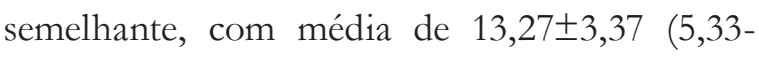
18). O domínio das relações sociais evidenciou

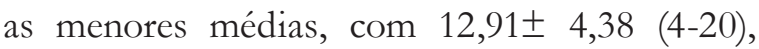
enquanto odomínio de meio ambienterepresentou as maiores médias dentre estes, com 14,38 $\pm 2,24$ $(9,71-19,43)$. O IGQV apresentou escore médio de 14,31 $\pm 4,15$ (6-20). O Alfa de Cronbach para todas as 26 questões do WHOQOL-bref foi de 0,91 , enquanto a consistência interna dos domínios físico, psicológico, relações sociais e meio ambiente foi de 0,$85 ; 0,85 ; 0,79$ e 0,71 , respectivamente; o IGQV evidenciou Alfa de 0,73. Encontrou-se correlação estatisticamente significativa entre o grau de dependência do idoso assistido e a sobrecarga percebida pelo cuidador familiar desse idoso $(\mathrm{r}=-0,281$, $\mathrm{p}=0,013)$, associando maiores escores no ZBI quando o idoso era mais dependente.

A análise da QV nos cuidadores por meio do instrumento WHOQOL-bref evidenciou correlação entre maior grau de dependência do idoso assistido e piores percepções no domínio físico ( $\mathrm{r}=0,279, \mathrm{p}=0,033)$, no domínio psicológico $(\mathrm{r}=0,483, \mathrm{p}=0,002)$, no domínio das relações sociais $(\mathrm{r}=0,356, \mathrm{p}=0,012)$ e no IGQV $(\mathrm{r}=0,331$, $\mathrm{p}=0,024)$. Não houve correlação na análise do domínio de meio ambiente. As diferenças entre as médias encontradas para cada um dos domínios e do IGQV segundo o grau de dependência do idoso podem ser visualizadas na tabela 3 . 
Tabela 3 - Percepção de qualidade de vida nos cuidadores de acordo com o instrumento WHOQOLbref, segundo o grau de dependência do idoso assistido em amostra de 45 cuidadores familiares. Curitiba-PR, 2008-2009.

\begin{tabular}{ccccccc}
\hline $\begin{array}{c}\text { Grau de } \\
\text { dependência }\end{array}$ & Físico & Psicológico & $\begin{array}{c}\text { Relações } \\
\text { sociais }\end{array}$ & $\begin{array}{c}\text { Meio } \\
\text { ambiente }\end{array}$ & IGQV $^{*}$ \\
\hline Dependente & média & 13,25 & 12,22 & 11,77 & 13,96 & 13,33 \\
& $\mathrm{n}$ & 30 & 30 & 30 & 30 & 30 \\
Independente & $\mathrm{dp}$ & 3,28 & 3,33 & 4,40 & 2,06 & 4,24 \\
& média & 15,42 & 15,37 & 15,20 & 15,23 & 16,26 \\
& $\mathrm{n}$ & 15 & 15 & 15 & 15 & 15 \\
Total & $\mathrm{dp}$ & 2,74 & 2,38 & 3,44 & 2,41 & 3,28 \\
& média & 13,98 & 13,27 & 12,91 & 14,38 & 14,31 \\
& $\mathrm{n}$ & 45 & 45 & 45 & 45 & 45 \\
& $\mathrm{dp}$ & 3,25 & 3,37 & 4,38 & 2,24 & 4,15 \\
\hline
\end{tabular}

*Índice Geral de Qualidade de Vida; dp=desvio-padrão.

Nota: a classificação do grau de dependência do idoso assistido foi determinada pela aplicação do Índice de Barthel, assumindo como independentes os idosos com pontuação superior a 60 neste instrumento.

A avaliação da correlação entre a $\mathrm{p}=0,004)$. Os cuidadores que não moram com percepção da QV dos cuidadores e as variáveis o idoso assistido $(\mathrm{n}=12)$ pontuaram média de sociodemográficas associadas ao cuidado 15,73 $\pm 2,79$ neste domínio, enquanto os pares identificou significância entre o domínio das que moram com o idoso entre dois e quatro anos relações sociais e a variável nominal "tempo $(n=10)$ pontuaram em média 10,44 $\pm 4,85$. morando junto com o idoso” (Kruskall-Wallis - 
Tabela 4 - Percepção de qualidade de vida nos cuidadores de acordo com o instrumento WHOQOLbref, conforme a percepção de sobrecarga pela escala Zarit Burden Interview, em amostra de 45 cuidadores familiares. Curitiba-PR, 2008-2009.

\begin{tabular}{lcccccc}
\hline Sobrecarga & Físico & Psicológico & $\begin{array}{c}\text { Relações } \\
\text { sociais }\end{array}$ & $\begin{array}{c}\text { Meio } \\
\text { ambiente }\end{array}$ & IGQV* \\
\hline Pequena & média & 16,42 & 15,66 & 16,16 & 16,71 & 17,50 \\
& $\mathrm{n}$ & 8 & 8 & 8 & 8 & 8 \\
Moderada & $\mathrm{dp}{ }^{\dagger}$ & 2,23 & 1,18 & 2,30 & 1,45 & 3,50 \\
& média & 14,14 & 14,43 & 13,40 & 14,28 & 14,80 \\
Moderada a & $\mathrm{n}$ & 20 & 20 & 20 & 20 & 20 \\
Severa & $\mathrm{dp}$ & 3,40 & 2,59 & 4,48 & 2,08 & 3,33 \\
& média & 12,57 & 10,57 & 10,19 & 13,42 & 12,00 \\
Severa & $\mathrm{n}$ & 14 & 14 & 14 & 14 & 14 \\
& $\mathrm{dp}$ & 2,97 & 3,57 & 4,13 & 1,95 & 4,64 \\
& média & 12,95 & 11,77 & 13,77 & 13,33 & 13,33 \\
& $\mathrm{n}$ & 3 & 3 & 3 & 3 & 3 \\
Total & $\mathrm{dp}$ & 2,81 & 2,77 & 2,03 & 2,70 & 3,05 \\
& $\mathrm{n}$ & 13,98 & 13,27 & 12,91 & 14,38 & 14,31 \\
& $\mathrm{n}$ & 45 & 45 & 45 & 45 & 45 \\
& $\mathrm{n}$ & 3,25 & 3,37 & 4,38 & 2,24 & 4,15 \\
\hline
\end{tabular}

*Índice Geral de Qualidade de Vida; † desvio-padrão.

Nota: a classificação do grau de sobrecarga inerente ao cuidado foi determinada de acordo com a classificação adotada por Oliveira e colaboradores. ${ }^{8}$

A análise bivariada identificou correlação entre as categorias de sobrecarga relacionadas ao cuidado e as seguintes características do cuidador: gênero ( $\mathrm{r}=-0,295, \mathrm{p}=0,024)$; estado civil ( $\mathrm{r}=-0,280, \mathrm{p}=0,031)$; tempo como cuidador $(\mathrm{r}=-0,309, \quad \mathrm{p}=0,019) ; \quad$ parentesco $\quad(\mathrm{r}=0,275$, $\mathrm{p}=0,034)$; grau de dependência funcional ( $\mathrm{r}=$ $0,281, p=0,031)$; qualidade de vida representada pelos domínios físico $(\mathrm{r}=-0,437, \mathrm{p}=0,001)$, psicológico $(\mathrm{r}=-0,564, \mathrm{p}<0,001)$, relações sociais $(\mathrm{r}=-0,422, \mathrm{p}=0,002)$, meio ambiente $(\mathrm{r}=-0,439$, $\mathrm{p}<0,001)$ e IGQV $(\mathrm{r}=-0,439, \mathrm{p}=0,001)$.
A regressão linear múltipla identificou forte associação entre a severidade da sobrecarga relacionada ao cuidado (escore total do ZBI) e a percepção de qualidade de vida para o domínio psicológico do WHOQOL-bref (IC 95\% -3,801 a -1,850), bem como para a variável "tempo como cuidador" (IC 95\% -12,849 a -5,196), com valor de $\mathrm{p}<0,001\left(\mathrm{R}^{2}=0,574\right)$. A figura 1 ilustra a confirmação de que a distribuição dos erros no modelo desta regressão linear múltipla segue uma distribuição normal. 


\section{Normal P-P Plot of Regression Standardized Residual \\ Dependent Variable: Sobrecarga relacionada ao cuidado (Zarit Burden Interview - Escore total)}

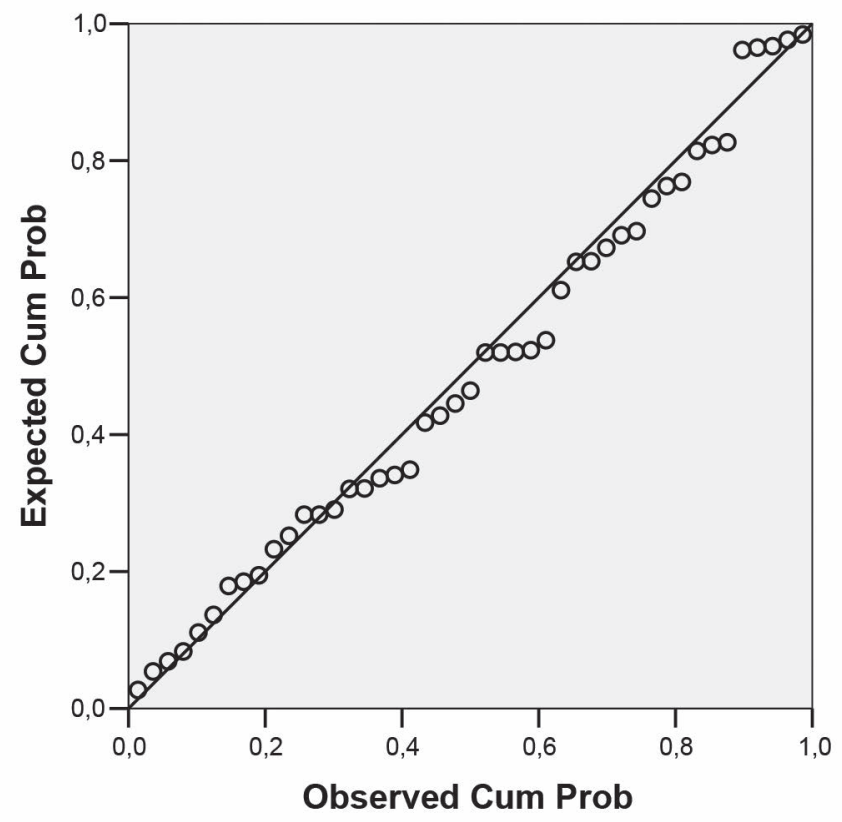

Figura 1 - Gráfico confirmando que a suposição de normalidade dos erros está satisfeita para esta regressão linear múltipla, em amostra de 45 cuidadores familiares. Curitiba-PR, 2008-2009.

\section{DISCUSSÃO}

O cuidador familiar é percebido como importante fonte de apoio para o enfrentamento da dependência. ${ }^{16}$ Reconhecer o perfil desses cuidadores e disponibilizar assistência profissional direcionada às demandas deste grupo pode contribuir não só para melhorar a qualidade do cuidado, mas também disponibilizar meios para garantir a preservação da saúde física e psicológica da família e, principalmente, do cuidador familiar. ${ }^{11,17}$

O perfil da amostra estudada identificou forte participação da mulher neste papel, com uma maioria de filhas casadas e/ou em união consensual, com média etária entre 50 e 60 anos, morando com o familiar idoso assistido.
Este perfil encontra forte respaldo na literatura, tendo sido o padrão encontrado por autores que investigaram cuidadores de idosos portadores de demência ${ }^{17-20}$ e acidente vascular cerebral, ${ }^{21,22}$ cuidadores entrevistados em ambiente ${\text { ambulatorial }{ }^{7,9} \text { e domiciliário }}^{3,5,11}$ ou assistidos pela Estratégia Saúde da Família, ${ }^{8,23,24}$ bem como estudos em outros países. ${ }^{25,26}$

A elevada proporção de cuidadores com nível superior de escolaridade (53\%), fato não replicado na maioria dos estudos com cuidadores familiares, 5,7,8,17-19,21,22,25,26 talvez seja reflexo da amostragem adotada pelo estudo, e pela característica urbana da população pesquisada. Apenas um levantamento ${ }^{20}$ realizado em Belo Horizonte com cuidadores participantes de grupos de apoio encontrou perfil de escolaridade semelhante, 
com 46,6\% das cuidadoras apresentando ensino superior completo; os autores também acreditam que a elevada escolaridade possa ser justificada por viés de seleção.

O tempo que o cuidador familiar dedicava ao cuidado foi superior a dez anos em pequena parcela da amostra $(11,11 \%)$, inferior à proporção encontrada em estudo com cuidadores de idosos atendidos entrevistados em nível ambulatorial na cidade de Campinas (33,3\%). ${ }^{7}$ A média de tempo que um cuidador familiar vem exercendo suas funções, porém, varia substancialmente de acordo com o tipo de delineamento e população estudada, sendo identificadas na literatura médias entre dois e três anos, ${ }^{10,24}$ quatro e sete anos, ${ }^{17,19,20}$ e nove anos ou mais. ${ }^{8,23}$

O nível médio de sobrecarga na amostra pesquisada foi semelhante ao encontrado em outros estudos envolvendo cuidadores familiares de idosos frágeis. ${ }^{7,820,23}$ A distribuição dos cuidadores de acordo com a classificação do grau de sobrecarga (tabela 2) evidenciou que a amostra estudada apresenta perfil semelhante ao descrito na literatura, ${ }^{7,20}$ com predomínio de casos classificados como sobrecarga moderada e moderada a severa, e poucos casos classificados como sobrecarga severa. Já conhecidas as associações teóricas entre dependência funcional e sobrecarga, ${ }^{6,24}$ e sendo alto o percentual de idosos dependentes na amostra, a elevada proporção de sobrecarga relacionada ao cuidado era esperada como resultado neste estudo.

Diferentemente de estudos ${ }^{8,23}$ que encontraram maiores escores na aplicação do WHOQOL-bref em cuidadores familiares nos domínios físico e de relações sociais, encontramos maiores pontuações no IGQV e no domínio de meio ambiente, à semelhança de outro autor. ${ }^{9} \mathrm{O}$ desempenho dos cuidadores na avaliação da QV pode ser fortemente influenciado pelo nível de escolaridade, mas estudos anteriores sugerem que muitas variáveis podem causar impacto nesta percepção, incluindo a sobrecarga relacionada ao cuidado, ${ }^{9}$ a idade do cuidador e a idade do idoso assistido, ${ }^{23}$ assim como a presença de doença no cuidador e seu estado civil. ${ }^{8}$

Ao pesquisar a correlação do grau de dependência funcional, da qualidade de vida dos cuidadores familiares e da sobrecarga relacionada ao cuidado, foi possível estabelecer o achado de correlação entre maior grau de comprometimento funcional e maiores níveis de sobrecarga no cuidador, à semelhança de outros autores. ${ }^{6,11,24} \mathrm{~A}$ qualidade de vida nos cuidadores foi pior quanto maior o grau de dependência, a exemplo de outros autores que utilizaram o mesmo instrumento,, 23 e percebida como pior quando a capacidade funcional do idoso esteve mais comprometida, mesmo quando aplicados outros instrumentos, como o Short Form 36 (SF-36). ${ }^{19}$

A análise bivariada identificou possíveis correlações para a severidade da sobrecarga relacionada ao cuidado (ZBI), quando analisadas as variáveis sociodemográficas em conjunto com as escalas estudadas para as seguintes variáveis: gênero e estado civil do cuidador, parentesco, dependência funcional, tempo como cuidador, e domínios físico, psicológico, de relações sociais, de meio ambiente e IGQV do WHOQOL-bref.

A literatura destaca alguns fatores como fortemente associados à sobrecarga familiar, como comportamentos problemáticos dos pacientes, perdas ocupacionais, financeiras e de suporte decorrentes da doença, ${ }^{6}$ ocupação do cuidador, prática de esportes, religiosidade e tempo na função como cuidador, ${ }^{20}$ cuidar de idosos mais velhos e com maior comprometimento cognitivo, ${ }^{11}$ bem como ser um cuidador mais velho. ${ }^{23}$

A aplicação da regressão linear múltipla identificou como fortemente relacionados a modificações no escore total do ZBI o tempo total dedicado ao cuidado e a pontuação do domínio psicológico do instrumento WHOQOL-bref para avaliação da qualidade 
de vida. Cuidadores familiares de pacientes dependentes frequentemente lidam com situações de dor física, restrições funcionais e comprometimento emocional que causam elevado desgaste emocional, ${ }^{19}$ causando forte impacto negativo nas percepções psicológicas e repercussões importantes nas percepções de sobrecarga. Estudos anteriores confirmam que a sobrecarga dos cuidados é um forte e relevante preditor de desconforto emocional. ${ }^{11}$ Do mesmo modo, a tendência de maior sobrecarga quanto maior o tempo dedicado à função de cuidador também foi confirmada por outros autores. ${ }^{6,20}$

Em resumo, em uma amostra de cuidadores familiares predominantemente feminina, casada e de elevada escolaridade, foram identificadas correlações estatisticamente significativas entre menor nível de sobrecarga relacionada ao cuidado e melhores percepções de qualidade de vida, bem como associação entre maior grau de dependência do idoso assistido e maior sobrecarga e QV menos satisfatórias. O tempo dedicado à função de cuidador e a percepção de qualidade de vida no domínio psicológico foram as variáveis que mais fortemente se associaram à sobrecarga.

Este estudo apresenta limitações quanto à generalização de seus achados. Diversas variáveis associadas à caracterização da relação de cuidado não foram estudadas, como o tempo diário dedicado ao cuidado, a disponibilidade de rede de apoio social, o tipo de atividade desenvolvida e a presença de comorbidades, depressão e ansiedade nos cuidadores e nos idosos. A variável "tempo como cuidador" foi coletada e analisada apenas como variável categórica, prejudicando a análise descritiva e reduzindo o poder estatístico de alguns testes. Do mesmo modo, a amostragem não probabilística e o tamanho da amostra reduzem a possibilidade de encontrar outras associações significativas nos cuidadores entrevistados, bem como a validade externa do estudo.

\section{CONCLUSÃO}

A sobrecarga relacionada ao cuidado é uma condição de risco cotidianamente vivenciada por diversos familiares que desempenham o papel de cuidador. É exatamente por meio de investigações que se conhecem os principais determinantes e fatores que podem contribuir para o desenvolvimento de sobrecarga e percepções negativas associadas ao cuidado. Este estudo encontrou correlação estatisticamente significativa entre o grau de dependência funcional do idoso e o nível de sobrecarga percebida pelo cuidador familiar $(r=-0,281, p=0,013)$ e piores percepções de qualidade de vida nos domínios físico, psicológico, de relações sociais e no índice geral de qualidade de vida (IGQV), quanto maior o grau de incapacidade física. Do mesmo modo, encontrou forte associação entre o tempo dedicado à função de cuidador e a percepção de qualidade de vida no domínio psicológico com a sobrecarga relacionada ao cuidado $\left(\mathrm{R}^{2}=0,574, \mathrm{p}<0,001\right)$.

Poucos estudos na literatura avaliaram a relação entre o grau de dependência funcional e os níveis de sobrecarga e qualidade de vida nos cuidadores. Intervenções individuais que disponibilizem orientações e educação sobre como realizar o cuidado de modo apropriado, sem agregar sobrecarga física e psicológica às tarefas diárias, aliadas a políticas públicas que viabilizem adequada rede social de apoio e suporte da atenção primária em saúde, só serão possíveis com o reconhecimento das características deste segmento. Do mesmo modo, reconhecer o perfil de cuidadores com elevada escolaridade é significativo: frente à redução no tamanho dos núcleos familiares e a falta de mão de obra qualificada para atender à demanda crescente de idosos dependentes morando no próprio domicílio, é provável que cada vez mais filhos, netos e cônjuges acabem se responsabilizando pelo cuidado domiciliário. 


\section{REFERÊNCIAS}

1. Camarano AA. Envelhecimento da população brasileira: uma contribuição demografica.In: Freitas EV, Py L, Neri AL, Cançado FAX, Gorzoni ML, Rocha SM, editores.. Tratado de Geriatria e Gerontologia. 2. ed. Rio de Janeiro: Guanabara Koogan; 2006. p. 88-105.

2. Monin JK, Schulz R. Interpersonal effects of suffering in older adult caregiving relationships. Psychol Aging 2009;24(3):681-95.

3. Vaingankar JA, Subramaniam M, Abdin E, He VY, Chong SA. "How much can I take?": predictors of perceived burden for relatives of people with chronic illness. Ann Acad Med Singapore 2012;41(5):212-20.

4. Langa KM, Chernew ME, Kabeto MU, Herzoq AR, Ofstedal MB, Willis RJ, et al. National estimates of the quantity and cost of informal caregiving for the elderly with dementia. J Gen Intern Med 2001;16(11):770-8.

5. Ferreira CG, Alexandre TS, Lemos ND. Fatores associados à qualidade de vida de cuidadores de idosos em assistência domiciliária. Saude Soc 2011;20(2):398-409.

6. Baptista BO, Beuter M, Girardon-Perlini NMO, Brondani CM, Budó MLD, Santos NO. A sobrecarga do familiar cuidador no âmbito domiciliar: uma revisão integrativa da literatura. Rev Gaucha Enferm 2012;33(1):147-56.

7. Oliveira DC, Carvalho GSF, Stella F, Higa CMH, D'Elboux MJ. Qualidade de vida e sobrecarga de trabalho em cuidadores de idosos em seguimento ambulatorial. Texto Contexto Enferm 2011;20(2):234-40.

8. Amendola F, Oliveira MAC, Alvarenga MRM. Qualidade de vida dos cuidadores de pacientes dependentes no programa de saúde da família. Texto Contexto Enferm 2008;17(2):266-72.

9. De Nardi T, Rigo JC, Brito M, Santos ELM, Brós AJG. Sobrecarga e percepção de qualidade de vida em cuidadores de idosos do Núcleo de Atendimento à Terceira Idade do Exército (Natiex). Rev Bras Geriatr Gerontol 2011;14(3):511-9.

10. Inouye K, Pedrazzani ES, Pavarini SCI. Octogenários e cuidadores: perfil sócio-demográfico e correlação da variável qualidade de vida. Texto Contexto Enferm 2008;17(2):350-7.
11. Gratao ACM, Vendrúscolo TRP,000 Talmelli LFS, Figueiredo LC, Santos JLF, Rodrigues RAP. Sobrecarga e desconforto emocional em cuidadores de idosos. Texto Contexto Enferm 2012;21(2):304-12.

12. Scazufca M. Brazilian version of the Burden Interview scale for the assessment of burden of care in carers of people with mental illnesses. Rev Bras Psiquiatr 2002;24(1):12-7.

13. Chachamovich E, Fleck MPA. Desenvolvimento do Whoqol-Bref. In: Fleck MPA, organizador. A avaliação de qualidade de vida: guia para profissionais da saúde. Porto Alegre: ArtMed; 2008. p.74-82.

14. Minosso JSM, Amendola F, Alvarenga MRM, Oliveira MAC . Validação, no Brasil, do Índice de Barthel em idosos atendidos em ambulatórios. Acta Paul Enferm 2010;23(2):218-23.

15. Quinn TJ, Langhorne P, Stott DJ. Barthel index for stroke trials: development, properties, and application. Stroke 2011;42(4):1146-51.

16. Inouye K, Pedrazzani ES, Pavarini SCI. Implicações da doença de Alzheimer na qualidade de vida do cuidador: um estudo comparativo. Cad Saude Pub 2010;26(5):891-9.

17. Borghi AC, Sassá AH, Matos PCB, Decesaro MN, Marcon SS. Qualidade de vida de idosos com doença de Alzheimer e de seus cuidadores. Rev Gaucha Enferm 2011;32(4):751-8.

18. Inouye K, Pedrazzani ES,Pavarini SCI, Toyoda CY. Perceived quality of life of elderly patients with dementia and family caregivers: evaluation and correlation. Rev Latino-Am Enferm 2009;17(2):187-93.

19. Pinto MF, Barbosa DA, Ferreti CEL, Souza LF, Fram DS, Belasco AGS. Qualidade de vida de cuidadores de idosos com doença de Alzheimer. Acta Paul Enferm 2009;22(5):652-7.

20. Silva CF, Passos VMA, Barreto SM. Frequência e repercussão da sobrecarga de cuidadoras familiares de idosos com demência. Rev Bras Geriatr Gerontol 2012;15(4):707-31.

21. McPherson CJ, Wilson KG, Chyurlia L, Leclerc C. The caregiving relationship and quality of life among partners of stroke survivors: a cross-sectional study. Health Qual Life Outcomes 2011;9(1):29. 
22. Bergström AL, Eriksson G, von Koch L, Tham K. Combined life satisfaction of persons with stroke and their caregivers: associations with caregiver burden and the impact of stroke. Health Qual Life Outcomes 2011;9:1.

23. Moreira PHB, Mafra SCT, Pereira ET, Silva VE. Qualidade de vida de cuidadores de idosos vinculados ao Programa Saúde da Família - Teixeiras, MG. Rev Bras Geriatr Gerontol 2011;14(3):433-40.

24. Uesugui HM, Fagundes DS, Pinho DLM. Profile and degree of dependency of the elderly and overload of their caregivers. Acta Paul Enferm 2011;24(5):685-98.
25. Pimenta GMF, Costa MASMC, Gonçalves LHT, Alvarez AM. Perfil do familiar cuidador de idoso fragilizado em convívio doméstico da grande Região do Porto, Portugal. Rev Esc Enferm USP 2009;43(3):609-14.

26. Rodríguez-Sánchez E, Pérez-Peñaranda A, LosadaBaltar A, Pérez-Arechaederra D, Gómez-Marcos MA, Patino-Alonso MC, et al. Relationships between quality of life and family function in caregiver. BMC Fam Pract 2011;12:19. 